\title{
EDITORIAL
}

\section{Biennial IUFRO Landscape Ecology Conference}

\author{
Conferencia bienal de Ecología de Paisaje de IUFRO
}

\author{
Cristian Echeverría \\ Chair, 2012 IUFRO Landscape Ecology Conference (www.iufrole2012.cl) \\ Co-Editor, Bosque \\ Universidad de Concepción, Facultad de Ciencias Forestales, Concepción, Chile, phone: (56 41) 2204936, \\ fax: (56-41) 2255164, cristian.echeverria@udec.cl
}

The recent Biennial Conference of the IUFRO Landscape Ecology Working Party 8.01.02, entitled, "Sustaining humans and forests in changing landscapes: forest, society and global change" was held in Concepción, Chile from the $2^{\text {nd }}$ to the $12^{\text {th }}$ of November, 2012. This Working Party is coordinated by Jiquan Chen (University of Toledo, Ohio, USA) and seeks to promote and facilitate the application of landscape ecology concepts in the policies and practices of forested landscapes worldwide. It also encourages communication and interaction among scientists who have an interest in landscape ecology and forestry. For this purpose, the Working Party has gathered in seven places across the world: Slovenia, the United States, Canada, Japan, Italy, China and two years ago, Portugal. This year, it was the first time that the Working Party gathered further south in the southern hemisphere, and in particular, in Latin America. The region offers new challenges to forest landscape ecology due to its tremendous heterogeneity of ecosystems and diverse socioeconomic conditions. South American forests represent a large fraction of the world's biodiversity and, in turn, are characterized by progressive deforestation and degradation.

During the 2012 Conference, we received diverse types of scientific contributions such as keynote speakers' plenaries, symposia, oral presentations, posters and short manuscripts for publication in an 'ISI' journal. The contributing topics ranged from forest landscape management to studies on spatial patterns and ecological processes including the effects of climate change and landscape planning. These studies were conducted in diverse, contrasting landscapes, from the subAntarctic forests in Cape Horn, Chile to the Siberian forests, including landscapes from Brazil to Australia and Turkey.

It is our major interest to promptly divulgate these works through publication in an open-access scientific journal that is widely known in Latin America. With this goal, the Issue 33 (3) of the Bosque Journal is dedicated to the publication of the short manuscripts accepted by the conference's scientific committee. The scientific committee was formed by Louis Iverson (Chair, USDA Forest Service, USA), João Azevedo (Instituto Politécnico de Bragança, Portugal), Jean Paul Metzger (Universidade de Sao Paulo, Brazil), Sandra Luque (IRSTEA, France), Ajith Perera (Ontario Ministry of Natural Resources, Canada), Guillermo Martinez Pastur (Centro Austral de Investigaciones Científicas, Argentina), Damasa Magcale-Macandog (University of the Philippines Los Banos, Philippine) and Jan Boegart (Universite Libre de Bruxelles, Belgium). The process of publication was coordinated by Laura Nahuelhual (Universidad Austral de Chile), who is a member of the conference's organizing committee. 


\title{
EDITORIAL
}

\section{Conferencia bienal de Ecología de Paisaje de IUFRO}

\author{
Biennial IUFRO Landscape Ecology Conference
}

\author{
Cristian Echeverría \\ Chair, 2012 IUFRO Landscape Ecology Conference (www.iufrole2012.cl) \\ Co-Editor, Bosque \\ Universidad de Concepción, Facultad de Ciencias Forestales, Concepción, Chile, phone: (56 41) 2204936,
} fax: (56-41) 2255164, cristian.echeverria@udec.cl

Entre el 2 y 12 de noviembre del presente año se celebró en Concepción, Chile, una nueva versión del congreso internacional IUFRO (International Union of Forest Organizations) del Grupo de Trabajo en Ecología de Paisaje 8.01.02, titulada Sustaining humans and forests in changing landscapes, forest, society and global change. Este grupo es coordinado por Jiquan Chen (Universidad de Toledo, Ohio, EE.UU.) y tiene como objetivo promover y facilitar la aplicación de los conceptos de la ecología de paisaje en políticas y prácticas de paisajes forestales a nivel mundial. También busca incentivar la comunicación e interacción entre científicos con interés en la ecología de paisaje y manejo forestal. Con este propósito, se han sostenido siete reuniones internacionales en diversos países, a saber, Eslovenia, Estados Unidos, Canadá, Japón, Italia, China y, hace dos años, en Portugal. En noviembre 2012, la reunión del Grupo de Trabajo se celebró por primera vez en el hemisferio austral y, en particular, en América Latina. Esta región ofrece enormes desafíos para la ecología de paisajes forestales, debido a su gran heterogeneidad de ecosistemas y diversas condiciones socioeconómicas. Los bosques de Sudamérica representan una parte importante de la biodiversidad del planeta y, a su vez, se caracterizan por sufrir una progresiva deforestación y degradación.

En el encuentro de Concepción, Chile, se recibieron diversos tipos de contribuciones científicas, tales como plenarias, simposios, comunicaciones orales, pósteres y manuscritos cortos para su publicación en una revista 'ISI'. Las temáticas variaron desde el manejo de paisajes forestales hasta el estudio de patrones espaciales y procesos ecológicos, pasando por los efectos del cambio climático y los cambios del paisaje. Estos trabajos fueron conducidos en áreas de estudio muy diferentes y contrastantes, desde los bosques sub-antárticos del Cabo de Hornos hasta los bosques del sur de Siberia, incluyendo los paisajes de Brasil, Australia y Turquía, entre otros.

Es nuestro interés dejar a disposición del público, lo antes posible, estos trabajos científicos, de texto completo y libre acceso, a través de una revista científica de dilatada trayectoria en América Latina. Por ello, el número 33(3) de la revista Bosque está dedicado a la publicación de los manuscritos aceptados por el comité científico del presente congreso. Este comité científico estuvo presidido por Louis Iverson (USDA Forest Service, EE.UU.) y conformado, además, por João Azevedo (Instituto Politécnico de Bragança, Portugal), Jean Paul Metzger (Universidade de Sao Paulo, Brasil), Sandra Luque (IRSTEA, Francia), Ajith Perera (Ontario Ministry of Natural Resources, Canadá), Guillermo Martínez Pastur (Centro Austral de Investigaciones Científicas, Argentina), Damasa Magcale-Macandog (University of the Philippines Los Banos, Filipinas) y Jan Boegart (Universite Libre de Bruxelles, Bélgica). El proceso de publicación estuvo coordinado por Laura Nahuelhual (Universidad Austral de Chile), quien fue miembro del comité organizador del presente congreso. 